\title{
Theoretical Study of Optical Fiber Raman Polarizers With Counterpropagating Beams
}

\author{
Victor V. Kozlov, Javier Nuño, Juan Diego Ania-Castañón, and Stefan Wabnitz, Member, IEEE
}

\begin{abstract}
The theory of two counter-propagating polarized beams interacting in a randomly birefringent fiber via the Kerr and Raman effects is developed and applied to the quantitative description of Raman polarizers in the undepleted regime. Here Raman polarizers, first reported by Martinelli et al. [6], are understood as Raman amplifiers operating in the regime in which an initially weak unpolarized beam is converted into an amplified fully polarized beam towards the fiber output. Three parameters are selected for the characterization of a Raman polarizer: the degree of polarization of the outcoming beam, its state of polarization, and its gain. All of these parameters represent quantities that are averaged over all random polarization states of the initially unpolarized signal beam. The presented theory is computer friendly and applicable to virtually all practically relevant situations, including the case of co-propagating beams, and in particular to the undepleted as well as the depleted regimes of the Raman polarizer.
\end{abstract}

Index Terms-Optical fibers, polarization, Raman scattering.

\section{INTRODUCTION}

$\mathbf{F}$ IBER optic Raman amplifiers constitute an integral part of many contemporary high-speed optical networks. Owing to their broad amplification bandwidth, Raman amplifiers successfully compete with erbium-doped-fiber amplifiers, [1]. By the nature of the process of stimulated Raman scattering (SRS) in silica fibers, Raman amplifiers are highly polarization-dependent: the gain coefficient reaches its maximum value whenever the state of polarization (SOP) of the signal is parallel to the pump SOP. Whereas the amplifier gain is reduced by two orders of magnitude if the two SOPs are orthogonal, [2]-[4]. In real fiber networks the fiber birefringence changes stochastically along a fiber span. The net effect of this stochastically varying birefringence is to greatly reduce the polarization dependence of Raman gain. As a result, the effective Raman gain

Manuscript received September 08, 2010; revised November 26, 2010, December 06, 2010; accepted December 06, 2010. Date of publication December 17, 2010; date of current version January 28, 2011. This work was carried out in the framework of the "Scientific Research Project of Relevant National Interest" (PRIN 2008) entitled "Nonlinear cross-polarization interactions in photonic devices and systems" (POLARIZON), and appeared also as a result of 2009 Italy-Spain integrated action "Nonlinear Optical Systems and Devices" (HI2008-0075). The wok of J. Nuño was supported in part by the Spanish Ministry of Science and Innovation through an FPI fellowship (TEC2008-05791).

V. V. Kozlov is with the Department of Information Engineering, University of Brescia, Brescia 25123, Italy, and with the Department of Physics, Saint Petersburg State University, Saint Petersburg 198504, Russia (e-mail: victor.kozlov@email.com).

J. Nuño and J. D. Ania-Castañón are with the Instituto de Optica, Consejo Superior de Investigaciones Cientificas (CSIC), Madrid 28006, Spain (e-mail: javier.nuno@io.cfmac.csic.es; juan.diego@io.cfmac.csic.es).

S. Wabnitz is with the Department of Information Engineering, University of Brescia, Brescia 25123, Italy (e-mail: stefano.wabnitz@ing.unibs.it).

Digital Object Identifier 10.1109/JLT.2010.2099644 reduces to its span-averaged value, which is equal to approximately half of its maximum value. Such averaging of the gain between two orthogonal SOPs is a favorable feature, since polarization-dependent effects are undesirable in most of the telecom applications which have been developed so far. The value of the polarization-mode dispersion (PMD) coefficient $D_{p}$ quantifies the degree of gain averaging, which appears to be rather efficient for $D_{p}>0.2 \mathrm{ps} / \sqrt{\mathrm{km}}$. For smaller values of $D_{p}$, the onset of polarization-dependent Raman gain is typically combated by scrambling the pump SOP.

In this paper our interest diverges from the common trend, as it focuses on the exploitation of the polarization dependence of Raman gain. Motivated by the recent developments of transmission protocols based on polarization multiplexing [5], we are interested in developing nonlinear devices for achieving alloptical and ultrafast polarization control. Thus, we are interested in the use of fiber with low values of the PMD coefficient, and do not perform the scrambling of the pump beam. Moreover, the stronger the polarization-dependent gain, the better the performance of our device - a Raman polarizer. These devices are Raman amplifiers that are being fed by a weak unpolarized light signal, and convert it into a powerful yet highly polarized light towards the output. Preliminary theoretical investigations of Raman polarizers and their proof-of-principle experiment, both in the co-propagating geometry, have been recently reported in [6]. This geometry has been analyzed in more details in the undepleted regime in [7] and in the depleted regime in [8].

Here we set our goal in developing a simple and physically transparent theory of Raman polarizers which is applicable to both co-propagating and counter-propagating geometries, and suitable for further use as a tool for analyzing the operation of these devices. Our goal is also to address the two main issues which are related to the performance of Raman polarizers. The first issue is the degree of polarization (DOP) of the outcoming signal, which is defined as

$$
\mathrm{DOP}=\frac{\sqrt{\left\langle S_{1}^{(s)}\right\rangle^{2}+\left\langle S_{2}^{(s)}\right\rangle^{2}+\left\langle S_{3}^{(s)}\right\rangle^{2}}}{S_{0}^{(s)}} .
$$

Here $S_{j}^{(s)}$ is $j$ th component of the Stokes vector of the signal beam, and $S_{0}^{(s)}$ its power. As such, the DOP is the average length of the output Stokes vector over the $N$ realizations of the signal SOP (labeled by index $i$ ) which are imposed at the input. We shall be dealing with scrambled beams, that is with a set of $N$ fully polarized beams, whose SOPs are uniformly (or randomly) distributed over the Poincaré sphere.

Given that the average DOP of the scrambled signal is zero initially, we say that our goal is reached if the DOP of the output beam is close to unity. In this case, we shall refer to such Raman 
amplifiers as ideal Raman polarizers. The second issue is provided by the output signal SOP; namely, its relation to the pump SOP. The DOP and SOP of the outcoming signal fully characterize the performance of Raman polarizers. The third important quantity is the value of gain of the Raman polarizer. We shall comment on this issue, too.

It is important to distinguish from the outset between conventional Raman amplifiers and Raman polarizers. Typical Raman amplifiers for telecom applications are long, often surpassing $10 \mathrm{~km}$, require pump powers of the order of $1 \mathrm{~W}$, and operate with relatively high values of the PMD coefficient. In contrast, Raman polarizers are shorter, requiring a fiber length of the order of $2 \mathrm{~km}$, pumped with high power light — of the order of $5 \mathrm{~W}$ and higher, and favor low values of the PMD coefficient. The last two characteristics substantially strengthen the polarization dependence of the gain, as we shall see from the subsequent analysis. Therefore, the operational regimes of Raman amplifiers and Raman polarizers are drastically different, thus preventing the results of the theory of Raman polarizers to become a trivial copy of the results already known from the theory of Raman amplifiers.

The description of polarization-dependent gain in randomly birefringent fibers is based on the vector theory of SRS, as it was developed for instance in [9]-[12]. Theories presented in [10], [11] bring analytical insights into the problem, but their applicability is limited to the regime in which the beat length of the fiber $L_{B}$ is substantially smaller than the birefringence correlation length $L_{c}: L_{B} \ll L_{c}$. Because of this restriction, those theories are not suitable for the proper description of Raman polarizers, for which the opposite inequality usually holds, see [7] and the analysis that follows. In contrast, the theory in [12] is most general and accurate, however it is resource-consuming in terms of computational time, as it requires $10^{3} \div 10^{4}$ longer integration time than the theory which is presented here. Moreover, since the theory of [12] is not formulated in terms of deterministic differential equations, it is rather difficult to extract from it physically transparent information about the role of the different processes ruling the overall polarization dynamics.

As a matter of fact our theory is essentially the generalization of one-beam theory of Wai and Menyuk in [13] to the case of two beams interacting not only via the Kerr but also via the Raman effect. It is close to the approach that was undertaken in [11], but it proceeds till the end with virtually no approximations. The only important requirement here is that $\min \left(L, L_{N L}\right) \gg L_{c}$, where $L$ is the total length of the fiber and $L_{N L}$ its nonlinear length. This inequality holds true for almost all practically relevant situations. Another assumption in our theory is that in the randomly varying birefringence tensor characterizing the fiber

$$
\Delta \boldsymbol{B}=\Delta \beta(\omega)\left(\cos \theta \boldsymbol{\sigma}_{3}+\sin \theta \boldsymbol{\sigma}_{1}\right)
$$

we shall only treat the orientation of the birefringence axis $\theta$ as a stochastic variable, while we keep the value of the birefringence $\Delta \beta$ as a constant (this is the so-called fixed modulus model). It is now a well established fact that the theory with $\theta$ as the only stochastic variable and the theory where both $\theta$ and $\Delta \beta$ are stochastic variables (random modulus model) both produce virtually identical results. In (2), $\sigma_{1}$ and $\sigma_{3}$ are usual Pauli matrices. Note that we define the birefringence beat length as $L_{B}=2 \pi / \Delta \beta$.

The property of the Raman polarizer to polarize light may look similar to the mechanism of polarization attraction inherent to the so-called lossless polarizers. These lossless polarizers are based on the effect of four-wave mixing (FWM) between two strong beams. Lossless polarizers convert virtually any input signal SOP into a set of SOPs which remain close vicinity of a particular SOP, as prescribed by the pump beam polarization. This mechanism of polarization attraction is conservative in nature: lossless polarizers repolarize light without any of the polarization-dependent losses which are inherent to conventional passive polarizers. Historically the effect of FWM-assisted polarization attraction has been reported first for isotropic fibers, both theoretically and experimentally in [14]-[17]. Recently the effect was extended to randomly birefringent fibers, [18]-[20], and high-birefringence and unidirectionally spun fibers, [21].

In contrast, the Raman polarizers which are of interest to us here are nonconservative in nature, and the process of repolarization is accompanied with polarization-dependent gain/loss, similarly to passive polarizers. The mechanism here is the preferred amplification of one particular SOP and the rejecting of other SOPs. Whereas lossless polarizers intelligently repolarize light by transforming the input SOP by the mechanism of the nonlinear polarization rotation. An interesting example of the simultaneous action of the FWM and Raman attraction mechanisms was reported in [22]. However, we found that in our case the FWM mechanism does not produce any sizable effect, and the Raman attraction mechanism is fully responsible for the repolarization of light.

\section{MODEL}

Starting from the most basic model of interaction of two fields within a Kerr- and Raman-active medium, whose tensorial response as it is relevant to fused silica is properly taken into account, see [10], we follow the lines of derivations that were outlined in [23] and formulate the equation of motion of the pump beam in the form

$$
\begin{aligned}
\pm & i \partial_{z} U_{p}+i \beta^{\prime}\left(\omega_{p}\right) \partial_{t} U_{p}=-\Delta \boldsymbol{B} U_{p} \\
& -\frac{1}{3} \gamma_{p p}\left[2\left(U_{p}^{*} \cdot U_{p}\right) U_{p}+\left(U_{p} \cdot U_{p}\right) U_{p}^{*}\right] \\
& -\frac{2}{3} \gamma_{p s}\left[\left(U_{s}^{*} \cdot U_{s}\right) U_{p}+\left(U_{s} \cdot U_{p}\right) U_{s}^{*}+\left(U_{p} \cdot U_{s}^{*}\right) U_{s}\right] \\
& +i \epsilon_{p} g_{0}\left(U_{p} \cdot U_{s}^{*}\right) U_{s} .
\end{aligned}
$$

Here $U_{p}=\left(U_{p x}, U_{p y}\right)^{T}$ is the field vector written in terms of normal polarization modes $e_{x}$ and $e_{y} \cdot \beta\left(\omega_{p}\right)$ is the propagation constant and $\beta^{\prime}\left(\omega_{p}\right)$ is its frequency derivative. The upper sign ("+") describes the configuration when the signal and pump beams propagate in the fiber in one direction (co-propagating geometry), while the lower sign ("-") is related to the situation when they propagate in opposite directions (counter-propagating geometry). The self-polarization modulation (SPM) coefficient $\gamma_{p p}$ is the usual nonlinear coefficient of silica, $\gamma$, calculated at the central frequency $\omega_{p}$ of the pump beam. Cross-polarization modulation (XPM) coefficient $\gamma_{p s}$ is also equal to $\gamma\left(\omega_{p}\right)$; $g_{0}$ is the Raman gain coefficient; $\epsilon_{p}=-\omega_{p} / \omega_{s}$. The equations for the signal beam and all coefficients are the same as above but with indexes $p$ and $s$ interchanged, and with $\epsilon_{s}=1$.

First, let us transform the (3) to the local axes of birefringence by performing the rotation of the field vector $U_{j}$ as

$$
\bar{U}_{j}=\left(\begin{array}{cc}
\cos \frac{\theta}{2} & \sin \frac{\theta}{2} \\
-\sin \frac{\theta}{2} & \cos \frac{\theta}{2}
\end{array}\right) U_{j}
$$


where $j=p, s$. Equation (3) is not altered by this transformation, the only difference being the change of the form of the birefringence tensor, which now becomes

$$
\begin{aligned}
& \overline{\Delta \boldsymbol{B}}\left(\omega_{p}\right)=\left(\begin{array}{cc}
\Delta \beta\left(\omega_{p}\right) & \mp \frac{i}{2} \theta_{z} \\
\pm \frac{i}{2} \theta_{z} & -\Delta \beta\left(\omega_{p}\right)
\end{array}\right) \\
& \overline{\Delta \boldsymbol{B}}\left(\omega_{s}\right)=\left(\begin{array}{cc}
\Delta \beta\left(\omega_{s}\right) & -\frac{i}{2} \theta_{z} \\
\frac{i}{2} \theta_{z} & -\Delta \beta\left(\omega_{s}\right)
\end{array}\right) .
\end{aligned}
$$

Here, $\theta_{z}$ is the derivative of $\theta$ with respect to $z$. It is different from zero owing to the random changes of orientation of the birefringence axes. Namely, the change of $\theta$ is driven by the white noise process: $\theta_{z}=g_{\theta}(z)$, with zero mean and $\left\langle g_{\theta}(z) g_{\theta}\left(z^{\prime}\right)\right\rangle=2 L_{c}^{-1} \delta\left(z-z^{\prime}\right)$, where $L_{c}$ is the birefringence correlation length, as mentioned in the Introduction.

In line with our assumption that $\min \left(L, L_{N L}\right) \gg L_{c}$, we separate the fast motion related to the rapid changes of $\theta$ from the slow motion that occurs on the scale of the nonlinear length. It is important to note that this separation is exact and yet does not involve any approximation. The approximation is to be made at a later stage. The required transformations read as

$$
\begin{aligned}
& V_{p}=\boldsymbol{T}_{p}(z) \bar{U}_{p}=\left(\begin{array}{cc}
a_{1} & a_{2} \\
-a_{2}^{*} & a_{1}^{*}
\end{array}\right) U_{p} \\
& V_{s}=\boldsymbol{T}_{s}(z) \bar{U}_{s}=\left(\begin{array}{cc}
b_{1} & b_{2} \\
-b_{2}^{*} & b_{1}^{*}
\end{array}\right) U_{s} .
\end{aligned}
$$

Here $V_{p}=\left(V_{p 1}, V_{p 2}\right)^{T}$ and $V_{s}=\left(V_{s 1}, V_{s 2}\right)^{T}$. Matrices $\boldsymbol{T}_{p}$ and $\boldsymbol{T}_{s}$ obey equations of motion

$$
\begin{aligned}
& \pm i \partial_{z} \boldsymbol{T}_{p}+\overline{\Delta \boldsymbol{B}}\left(\omega_{p}\right) \boldsymbol{T}_{p}=0 \\
& i \partial_{z} \boldsymbol{T}_{\boldsymbol{s}}+\overline{\Delta \boldsymbol{B}}\left(\omega_{s}\right) \boldsymbol{T}_{s}=0 .
\end{aligned}
$$

These transformations eliminate the birefringence terms from the equations of motion of $V_{p}$ and $V_{s}$ and bring about a vast number of cubic terms composed of different combinations of $V_{p 1}, V_{p 2}, V_{s 1}, V_{s 2}$ and their complex conjugates. Factors in front of these terms are products of two coefficients of the form $u_{m} u_{n}$, or $u_{m}^{*} u_{n}$, or $u_{m}^{*} u_{n}^{*}$, where $m, n=1, \ldots, 14$. Products with $m=n$ we shall call self-products, while with $m \neq n$ cross-products. Here, $u_{1}=\left|a_{1}\right|^{2}-\left|a_{2}\right|^{2}, u_{2}=-\left(a_{1} a_{2}+a_{1}^{*} a_{2}^{*}\right)$, $u_{3}=i\left(a_{1} a_{2}-a_{1}^{*} a_{2}^{*}\right), u_{4}=2 a_{1} a_{2}^{*}, u_{5}=a_{1}^{2}-a_{2}^{* 2}, u_{6}=$ $-i\left(a_{1}^{2}+a_{2}^{* 2}\right), u_{7}=a_{1}^{*} b_{1}-a_{2} b_{2}^{*}, u_{8}=-\left(b_{1} a_{2}+b_{2}^{*} a_{1}^{*}\right), u_{9}=$ $i\left(b_{1} a_{2}-a_{1}^{*} b_{2}^{*}\right), u_{10}=-i\left(a_{1}^{*} b_{1}+a_{2} b_{2}^{*}\right), u_{11}=a_{1} b_{2}^{*}+b_{1} a_{2}^{*}$, $u_{12}=a_{1} b_{1}-a_{2}^{*} b_{2}^{*}, u_{13}=-i\left(a_{1} b_{1}+a_{2}^{*} b_{2}^{*}\right), u_{14}=i\left(a_{1} b_{2}^{*}-\right.$ $\left.a_{2}^{*} b_{1}\right)$.

In the thus obtained equations of motion for $V_{p}$ and $V_{s}$ we perform the ensemble average (over realizations of the random process that describes the linear birefringence). Thus, we write $\left\langle u_{m} u_{n}\right\rangle$ instead of $u_{m} u_{n}$. This change holds true only in the limit when the stochastic variations are faster than the nonlinear beam evolution. This is exactly the place in the derivation where our single approximation comes into play. At this point we also need to apply the ergodic theorem

$$
\langle f\rangle=\lim _{z \rightarrow \infty} \frac{1}{z} \int_{0}^{z} d z^{\prime} f\left(z^{\prime}\right)
$$

Our goal is to calculate ensemble averages of all necessary self- and cross-products, and on this way we may complete the derivation of the differential equations for $V_{p}$ and $V_{s}$.

The equations of motion for $u_{n}$ with $n=1, \ldots, 14$ can be easily formulated basing ourselves on (9) and (10). As these equations are linear, in order to find an ensemble average of any function of these coefficients (in our case pair products) we need to construct a generator. We refer to the Appendix in [13] for details of this procedure, and only give here the final result. With this generator we are able to formulate the equations of motion for the ensemble averages of the products of the coefficients. Thus, the solutions to the equations of motion

$$
\begin{aligned}
& \partial_{z} G_{1}=-2 L_{c}^{-1}\left(G_{1}-G_{2}\right), \\
& \partial_{z} G_{2}=2 L_{c}^{-1}\left(G_{1}-G_{2}\right) \mp 4 \Delta \beta\left(\omega_{p}\right) G_{4} \\
& \partial_{z} G_{3}= \pm 4 \Delta \beta\left(\omega_{p}\right) G_{4} \\
& \partial_{z} G_{4}=-L_{c}^{-1} G_{4} \pm 2 \Delta \beta\left(\omega_{p}\right)\left(G_{2}-G_{3}\right)
\end{aligned}
$$

yield the result for the self-products

$$
\left\{\left\langle u_{1}^{2}\right\rangle,\left\langle u_{2}^{2}\right\rangle,\left\langle u_{3}^{2}\right\rangle\right\},\left\{\left\langle\operatorname{Re}^{2}\left(u_{4}\right)\right\rangle,\left\langle\operatorname{Re}^{2}\left(u_{5}\right)\right\rangle,\left\langle\operatorname{Re}^{2}\left(u_{6}\right)\right\rangle\right\},
$$

and $\left\{\left\langle\operatorname{Im}^{2}\left(u_{4}\right)\right\rangle,\left\langle\operatorname{Im}^{2}\left(u_{5}\right)\right\rangle,\left\langle\operatorname{Im}^{2}\left(u_{6}\right)\right\rangle\right\}$, if we associate them with $\left\{G_{1}, G_{2}, G_{3}\right\}$ with initial conditions given as $(1,0,0),(0$, $1,0)$, and $(0,0,1)$, respectively.

The remaining self-products

$$
\begin{aligned}
& \left\{\left\langle\operatorname{Re}^{2}\left(u_{7}\right)\right\rangle,\left\langle\operatorname{Re}^{2}\left(u_{8}\right)\right\rangle,\left\langle\operatorname{Re}^{2}\left(u_{9}\right),\left\langle\operatorname{Re}^{2}\left(u_{10}\right)\right\rangle\right\},\right. \\
& \left\{\left\langle\operatorname{Im}^{2}\left(u_{7}\right)\right\rangle,\left\langle\operatorname{Im}^{2}\left(u_{8}\right)\right\rangle,\left\langle\operatorname{Im}^{2}\left(u_{9}\right),\left\langle\operatorname{Im}^{2}\left(u_{10}\right)\right\rangle\right\},\right. \\
& \left\{\left\langle\operatorname{Re}^{2}\left(u_{11}\right)\right\rangle,\left\langle\operatorname{Re}^{2}\left(u_{12}\right)\right\rangle,\left\langle\operatorname{Re}^{2}\left(u_{13}\right),\left\langle\operatorname{Re}^{2}\left(u_{14}\right)\right\rangle\right\},\right.
\end{aligned}
$$

and

$$
\left\{\left\langle\operatorname{Im}^{2}\left(u_{11}\right)\right\rangle,\left\langle\operatorname{Im}^{2}\left(u_{12}\right)\right\rangle,\left\langle\operatorname{Im}^{2}\left(u_{13}\right),\left\langle\operatorname{Im}^{2}\left(u_{14}\right)\right\rangle\right\},\right.
$$

can be found from the equations

$$
\begin{aligned}
& \partial_{z} G_{1}=-2 L_{c}^{-1}\left(G_{1}-G_{2}\right)+2 \Delta_{ \pm} G_{5} \\
& \partial_{z} G_{2}=2 L_{c}^{-1}\left(G_{1}-G_{2}\right)-2 \Delta_{ \pm} G_{6} \\
& \partial_{z} G_{3}=2 \Delta_{ \pm} G_{6} \\
& \partial_{z} G_{4}=-2 \Delta_{ \pm} G_{5} \\
& \partial_{z} G_{5}=\Delta_{ \pm}\left(G_{4}-G_{1}\right)-L_{c}^{-1} G_{5} \\
& \partial_{z} G_{6}=\Delta_{ \pm}\left(G_{2}-G_{3}\right)-L_{c}^{-1} G_{6}
\end{aligned}
$$

when we associate them with $\left\{G_{1}, G_{2}, G_{3}, G_{4}\right\}$, with initial conditions as $(1,0,0,0),(0,0,0,1),(0,1,0,0)$, and $(0,0,1,0)$, respectively. Here $\Delta_{ \pm} \equiv\left[ \pm \Delta \beta\left(\omega_{p}\right)-\Delta \beta\left(\omega_{s}\right)\right]$.

In order to find the cross-products we constructed appropriate generators and found that all the cross-products that are of interest to us turn out to be equal to zero. Similarly, terms of the form $\operatorname{Re}\left(u_{n}\right) \operatorname{Im}\left(u_{n}\right)$ also vanish. Thus, many SPM, XPM, and Raman terms in the final equations of motion disappear. The thus found equations of motion for the fields are conveniently formulated in Stokes space. They read as

$$
\begin{aligned}
\left( \pm \partial_{z}\right. & \left.+\beta^{\prime}\left(\omega_{p}\right) \partial_{t}\right) S^{(p)} \\
= & \gamma\left(\omega_{p}\right)\left(S^{(p)} \times \boldsymbol{J}_{S}^{(p)}(z) S^{(p)}+S^{(p)} \times \boldsymbol{J}_{X}(z) S^{(s)}\right) \\
& +\epsilon_{p} g_{0}\left(S_{0}^{(s)} J_{R 0} S^{(p)}+S_{0}^{(p)} \boldsymbol{J}_{R}(z) S^{(s)}\right) \\
\left(\partial_{z}+\right. & \left.\beta^{\prime}\left(\omega_{s}\right) \partial_{t}\right) S^{(s)} \\
= & \gamma\left(\omega_{s}\right)\left(S^{(s)} \times \boldsymbol{J}_{S}^{(s)}(z) S^{(s)}+S^{(s)} \times \boldsymbol{J}_{X}(z) S^{(p)}\right) \\
& +g_{0}\left(S_{0}^{(p)} J_{R 0} S^{(s)}+S_{0}^{(s)} \boldsymbol{J}_{R}(z) S^{(p)}\right) .
\end{aligned}
$$


Here $S^{(j)}$ is a three-component vector: $S^{(j)}=\left(S_{1}^{(j)}, S_{2}^{(j)}, S_{3}^{j}\right)$, whose components are defined as $S_{1}^{j}=V_{j 1} V_{j 2}^{*}+V_{j 1}^{*} V_{j 2}, S_{2}^{j}=$ $i\left(V_{j 1}^{*} V_{j 2}-V_{j 1} V_{j 2}^{*}\right), S_{3}^{j}=\left|V_{j 1}\right|^{2}-\left|V_{j 2}\right|^{2}$, where $j=p, s$. It is instructive to formulate separate equations for the powers of the signal and pump beams, which may be defined through the components of the Stokes vectors as $S_{0}^{(j)}=\left(S_{1}^{(j)^{2}}+S_{2}^{(j)^{2}}+\right.$ $\left.S_{3}^{(j)^{2}}\right)^{1 / 2}$

$$
\begin{aligned}
\left( \pm \partial_{z}\right. & \left.+\beta^{\prime}\left(\omega_{p}\right) \partial_{t}\right) S_{0}^{(p)} \\
= & \epsilon_{p} g_{0}\left(J_{R 0} S_{0}^{(s)} S_{0}^{(p)}+J_{R 1}(z) S_{1}^{(s)} S_{1}^{(p)}\right. \\
& \left.+J_{R 2}(z) S_{2}^{(s)} S_{2}^{(p)}+J_{R 3}(z) S_{3}^{(s)} S_{3}^{(p)}\right) \\
\left(\partial_{z}+\right. & \left.\beta^{\prime}\left(\omega_{s}\right) \partial_{t}\right) S_{0}^{(s)} \\
= & g_{0}\left(J_{R 0} S_{0}^{(s)} S_{0}^{(p)}+J_{R 1}(z) S_{1}^{(s)} S_{1}^{(p)}\right. \\
& \left.+J_{R 2}(z) S_{2}^{(s)} S_{2}^{(p)}+J_{R 3}(z) S_{3}^{(s)} S_{3}^{(p)}\right) .
\end{aligned}
$$

Matrices in (22) and (23) are all diagonal with elements $\boldsymbol{J}_{R}=\operatorname{diag}\left(J_{R 1}, J_{R 2}, J_{R 3}\right), \boldsymbol{J}_{X}=\operatorname{diag}\left(J_{X 1}, J_{X 2}, J_{X 3}\right)$, $\boldsymbol{J}_{S}=\operatorname{diag}\left(J_{S 1}, J_{S 2}, J_{S 3}\right)$. These elements are different for the counter-propagating and the co-propagating interaction geometries.

In order to complete our theory, we need to express all elements in these matrices in terms of ensemble averages of selfproducts:

$$
\begin{aligned}
J_{R 1} & =\left\langle\operatorname{Re}\left(u_{14}^{2}-u_{10}^{2}\right)\right\rangle \\
J_{R 2} & =-\left\langle\operatorname{Re}\left(u_{14}^{2}+u_{10}^{2}\right)\right\rangle \\
J_{R 3} & =-\left\langle\left|u_{14}\right|^{2}-\left|u_{10}\right|^{2}\right\rangle \\
J_{X 1} & =\frac{2}{3}\left\langle\operatorname{Re}\left(u_{10}^{2}+u_{13}^{2}-u_{9}^{2}-u_{14}^{2}\right)\right\rangle \\
J_{X 2} & =\frac{2}{3}\left\langle\operatorname{Re}\left(u_{10}^{2}+u_{14}^{2}-u_{9}^{2}-u_{13}^{2}\right)\right\rangle \\
J_{X 3} & =\frac{2}{3}\left\langle\left|u_{9}\right|^{2}+\left|u_{14}\right|^{2}-\left|u_{13}\right|^{2}-\left|u_{10}\right|^{2}\right\rangle \\
J_{S 1} & =\frac{1}{3}\left\langle\operatorname{Re}\left(u_{6}^{2}\right)\right\rangle \\
J_{S 2} & =-\frac{1}{3}\left\langle\operatorname{Re}\left(u_{6}^{2}\right)\right\rangle \\
J_{S 3} & =\frac{1}{3}\left[3\left\langle u_{3}^{2}\right\rangle-1\right]
\end{aligned}
$$

and also $J_{R 0}=\left\langle\left|u_{10}\right|^{2}+\left|u_{14}\right|^{2}\right\rangle$. Note that our model reduces to the one-beam theory of Wai and Menyuk when coefficients $u_{7}$ through $u_{14}$ are set to zero.

\section{Physical CONSIDERATIONS}

Model equations (22) and (23) represent the central finding of our paper. These equations describe vector Raman amplification under rather general conditions. The SPM, XPM, and Raman matrices contain $z$-dependent elements on their diagonals, whose values are expressed in terms of the self-product coefficients in (26)-(34), while the coefficients themselves are found as the solutions of (12)-(15) and (16)-(21).

We are about to apply these model equations to the problem of Raman polarizers. Before treating this specific case, some comment about the Raman matrix is in order. It is commonly accepted that the net effect of polarization-dependent gain is the

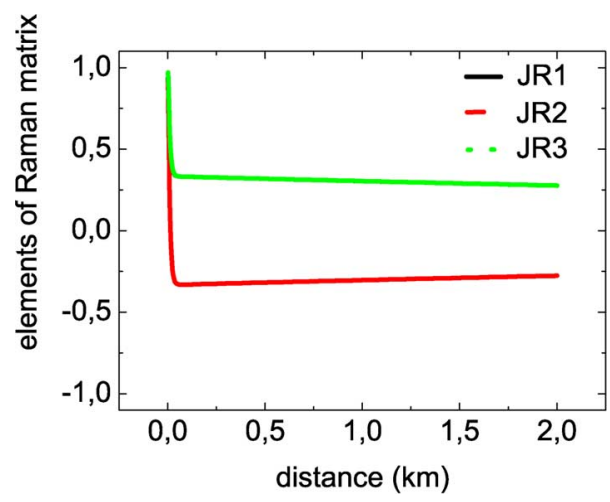

Fig. 1. Elements of the Raman matrix $J_{R}=\operatorname{diag}\left(J_{R 1}, J_{R 2}, J_{R 3}\right)$ ( $J_{R 1}$-black solid, $J_{R 2}$-red dashed, and $J_{R 3}$ - green dotted) as function of distance in the fiber for $L_{B}\left(\omega_{p}\right)=50 \mathrm{~m}$ and $L_{c}=1 \mathrm{~m}$. (Note that the black solid and green dotted curves nearly coincide, i.e., $J_{R 1}=J_{R 3}$.

attraction of the signal SOP to the pump SOP. While this is certainly the case for isotropic fibers, an extension of this idea to the case of randomly birefringent fibers should be undertaken with caution. As a matter of fact, we found that in general in randomly birefringent fibers the signal SOP is not attracted to the pump SOP. In order to demonstrate this, the most straightforward way is to analyze (25) for the power of the signal beam. For isotropic fibers, we get $J_{R 1}=J_{R 2}=J_{R 3}=1$, so that parallel signal and pump SOPs provide the maximally possible gain.

When we turn to the case of randomly birefringent fibers, we find that the elements of the Raman matrix are not always equal to each other in the co-propagating geometry, as it was demonstrated in [7]. Moreover, these elements are never equal to each other in the counter-propagating geometry, as it is exemplified in Fig. 1. As can be seen in this figure, the Raman matrix elements are not only different from each other but they can also be negative.

Although in general the signal SOP is not attracted to the pump SOP, the analysis of the co-propagating configuration in [7] shows that in the regime when Raman amplifiers act as ideal Raman polarizers the output signal SOP is always almost perfectly aligned with the pump SOP. For this regime, the polarization properties of the randomly birefringent fibers are virtually the same as in the case of isotropic fibers.

On the other hand in the counter-propagating geometry the situation is totally different. In the regime when Raman amplifiers act as ideal Raman polarizers, the typical relation between the diagonal elements of the Raman matrix is as shown in Fig. 1. Thus, the first and the third components of the signal Stokes vector are attracted to the corresponding components of the pump Stokes vector, while the second components are repelled from each other. Therefore, whenever the pump SOP contains an appreciable admixture of the circular polarization, the signal SOP is never attracted to the pump SOP. In the next section we suggest a simple rule with a wide range of applicability on how to determine the output signal SOP.

The polarization-dependent gain is quantified by the values of the diagonal elements of the Raman matrix. The larger these values, the better the performance of the Raman polarizers. In fibers with high PMD values the diagonal elements quickly vanish near the input fiber end already. For smaller PMD values the $z$-dependent elements of the Raman matrix keep 
appreciable values across the whole fiber span, as demonstrated in Fig. 1. Indeed, low PMD fibers are good candidates for the implementation of Raman polarizers.

The elements of the XPM matrix exhibit a similar dependence upon the PMD coefficient and have a magnitude which is comparable to that of the diagonal elements of the Raman matrix. Nevertheless the nonlinear polarization rotation which is due to the XPM interaction is very weak, and it has virtually no effect on the performance of Raman polarizers operating in the undepleted regime.

The SPM effect for the signal beam has also no impact on the performance of Raman polarizers. First, this is because the diagonal elements of the SPM matrix $\boldsymbol{J}_{\boldsymbol{S}}^{(s)}$ vanish on first $100 \mathrm{~m}$ of the fiber for the practically relevant range of beat lengths and correlation lengths, a range that we define as follows: $0.001<$ $L_{B}<0.1$ and $0.0001<L_{c}<0.05$, which is given here in $\mathrm{km}$. Second, because the signal beam is too weak to experience a significant nonlinear self-interaction, especially near its input end.

In contrast, the SPM effect can in principle be sizable for the pump beam. Given that the pump power is relatively high $(8 \mathrm{~W}$ in our simulations), even the first $100 \mathrm{~m}$ are enough to perturb the pump SOP. However, these perturbations remain relatively small (of the order of $0.1 \%$ ) for a pump power as high as $8 \mathrm{~W}$.

\section{NUMERICAL RESULTS}

We performed extensive simulations in a configuration where the pump and signal beams counter-propagate through a $2 \mathrm{~km}$ long fiber, and the pump power is set to $8 \mathrm{~W}$ at $z=L$. The input power of the signal beam is set to be equal to $0.1 \mathrm{~mW}$, which is low enough to ensure that Raman amplification occurs in the undepleted regime for all $z$ and the entire range of fiber parameters. We varied the beat length and the correlation length within the range that is defined above. The signal wavelength is $1.55 \mu \mathrm{m}$, while the pump frequency is upshifted by $13.2 \mathrm{THz}$. We followed two quantities of interest at the output end of the fiber at $z=L$ as function of the beat and the correlation lengths. The first such quantity is the DOP $(D)$ of the signal beam, which is the central characteristic of Raman polarizers. Starting with an unpolarized signal beam (with $D=0$ ), we aim at finding such parameter regimes for which the outcoming signal beam has DOP close to unity. The unpolarized signal beam is modeled in the Stokes space as an ensemble of beams with $N \sim 5000$ SOPs that are uniformly distributed over the entire Poincaré sphere. On the other hand the pump is a polarized beam whose SOP is varied as detailed below. The second quantity of interest is the so-called alignment parameter, which is defined as

$$
A_{\uparrow \downarrow} \equiv \frac{\left\langle S_{1}^{(s)} S_{1}^{(p)}-S_{2}^{(s)} S_{2}^{(p)}+S_{3}^{(s)} S_{3}^{(p)}\right\rangle}{S_{0}^{(s)} S_{0}^{(p)}}
$$

where the angle brackets indicate average over all $N$ realizations of the SOPs of the input scrambled signal beam. This quantity characterizes the average orientation of the signal SOP with respect to the pump SOP. Note that in the copropagating geometry the appropriate alignment parameter reads as [7]

$$
A_{\uparrow \uparrow} \equiv \frac{\left\langle S_{1}^{(s)} S_{1}^{(p)}+S_{2}^{(s)} S_{2}^{(p)}+S_{3}^{(s)} S_{3}^{(p)}\right\rangle}{S_{0}^{(s)} S_{0}^{(p)}} .
$$
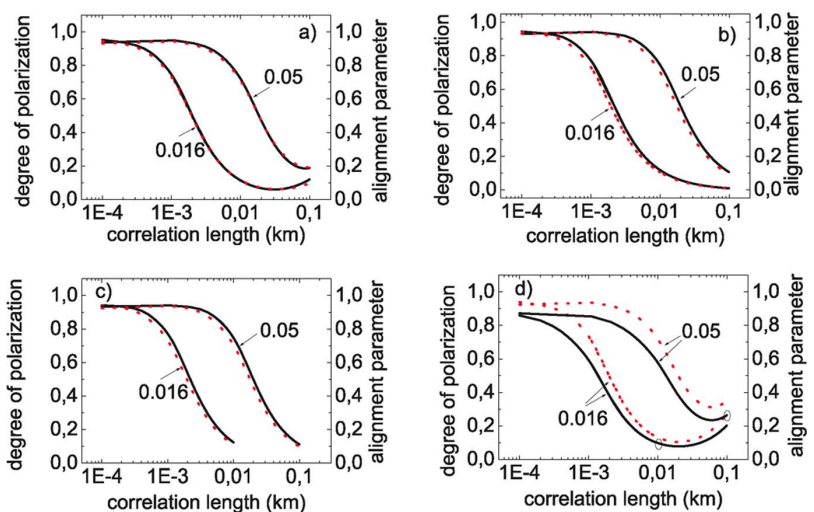

Fig. 2. DOP of the signal beam (black, solid) and alignment parameter $A_{\uparrow \downarrow}$ (red, dotted) as function of correlation length $L_{c}$ for four pump SOPs: (a) $3^{-1 / 2}(1,1,1)$; (b) $(1,0,0)$; (c) $(0,1,0)$; (d) $(0,0,1)$. The value of the birefringence length $L_{B}\left(\omega_{p}\right)$ is indicated on the plots in $\mathrm{km}$. Two circles on plot $d$ ) indicate one (of infinitely many) pair of points with equal PMD coefficients. Other parameters are: input signal power $0.1 \mathrm{~mW}$, input pump power $8 \mathrm{~W}$, Raman gain $g_{0}=0.6(\mathrm{~W} \cdot \mathrm{km})^{-1}$, nonlinearity parameter $\gamma=1(\mathrm{~W} \cdot \mathrm{km})^{-1}$, linear fiber losses $\alpha=0.2 \mathrm{~dB} / \mathrm{km}$, total length of the fiber $L=2 \mathrm{~km}$.
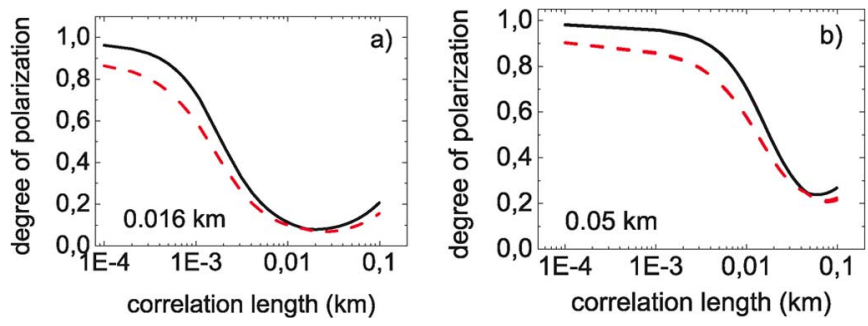

Fig. 3. DOP of the signal beam for two pump SOPs as function of the correlation length $L_{c}$ for the value of the birefringence length (a) $L_{B}=0.016 \mathrm{~km}$ and (b) $L_{B}=0.05 \mathrm{~km}$. Other parameters are the same as in Fig. 2. These two pump SOPs are those which maximize (black, solid) and minimize (red, dashed) the DOP of the output signal beam.

This alignment parameter is nothing but an average cosine of the angle between the pump and signal SOPs in Stokes space.

We start with the characterization of the performance of the Raman polarizer in the counter-propagating geometry by following the $D$ and $A_{\uparrow \downarrow}$ for four different SOPs of the pump beam and two values of the beat length, as a function of the correlation length. The corresponding results are shown in the four panels of Fig. 2. All four plots demonstrate that small correlation lengths and large beat lengths favor the efficiency of Raman polarizers. In the first three plots the maximum DOP is 0.94 , while on the fourth plot it is of only 0.84 . Such large difference in the maximal achievable DOPs gives a hint that the performance of Raman polarizers is rather sensitive to the choice of the pump SOP.

How sensitive is this dependence is demonstrated by the two panels of Fig. 3. For generating each point on these plots we took 256 realizations of the pump SOPs, and for each of this SOP we followed the signal DOP as a function of the correlation length. We plot only those two realizations which provide maximum and minimum DOP. From the analysis of these plots we may conclude that from the viewpoint of efficient Raman polarizers only a limited range of pump SOPs leads to a good performance, while the rest of pump SOPs perform poorly. The particular SOP which maximizes the DOP depends on both the correlation and the beat length values. 

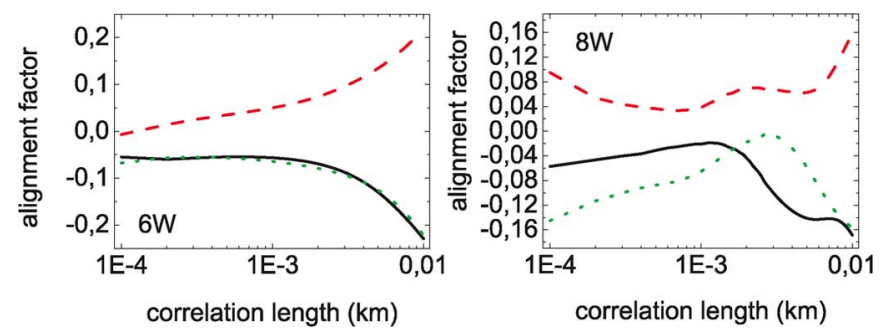

Fig. 4. Alignment factors defined in (37): $A_{\uparrow \downarrow}^{(1)}$ (black solid); $A_{\uparrow \downarrow}^{(2)}$ (red dashed); $A_{\uparrow \perp}^{(3)}$ (green dotted), as function of the correlation length for $L_{B}=0.05 \mathrm{~km}$ and for two values of the pump power: (a) $6 \mathrm{~W}$; (b) $8 \mathrm{~W}$.

Note that in all our numerical simulations we also take into account linear fiber loss $(\alpha)$. Also note that we set the Raman gain coefficient to zero in order to see if in the present repolarization results there is any effect of polarization attraction which is due to the lossless FWM mechanism. In such case we did not observe neither the alignment of the signal beam, nor the DOP different from zero. This observation indicates that the FWM mechanism does not play any role in our setup.

Coming back to the case of a nonzero Raman gain coefficient, we see that in all of the four plots of Fig. 2 we may observe that the alignment parameter is relatively close to unity whenever the DOP is more than 0.9. From this result we draw the conclusion that on average the SOP of the outcoming signal beam is related to the pump SOP as dictated by the alignment parameter that was introduced in (35). A more detailed information on the signal SOP is available when basing ourselves on the following definitions of the alignment factors

$$
A_{\uparrow \downarrow}^{(i)}=\left\langle\frac{S_{i}^{(s)}}{S_{0}^{(s)}}-\frac{S_{i}^{(p)}}{S_{0}^{(p)}}\right\rangle
$$

which are calculated at the Raman polarizer output $z=L$, here $i=1,2,3$. These expressions quantify the pairwise proximity of the components of the signal and pump Stokes vectors. The dependence of the alignment factors upon the correlation length for $L_{B}=50 \mathrm{~m}$ and for two values of the pump power is plotted in Fig. 4. One may observe that the output signal SOP depends on the pump power. Therefore, signal polarization stabilization may be deteriorated by relative intensity noise of the pump laser source.

Another important issue is the selection of fibers for Raman polarizers. Usually, this selection relies on the value of the PMD coefficient. In most practical situations, this coefficient fully characterizes a randomly birefringent fiber. However, in some cases the knowledge of this parameter only is not sufficient for making a conclusion on the performance of Raman polarizers, a fact that was first noticed in [7]. For example the plot in Fig. 2(d) demonstrates that two fibers with equal PMD coefficients lead to Raman polarizers with rather different performances: in one case $D \approx 0.26$, while in the other case it is 0.1 only. In the regime of ideal Raman polarizers (with DOP $>0.9$ ) and in the considered parameter range, the PMD coefficient is indeed a reliable characteristic, in the sense that fibers with equal PMD coefficients provide Raman polarizers with similar performances. Nevertheless, it is often desirable to consider the beat length and the correlation length separately, as we do in this study, rather than unite them under the single PMD coefficient. Note that for $L \gg L_{c}$, which is always the case for

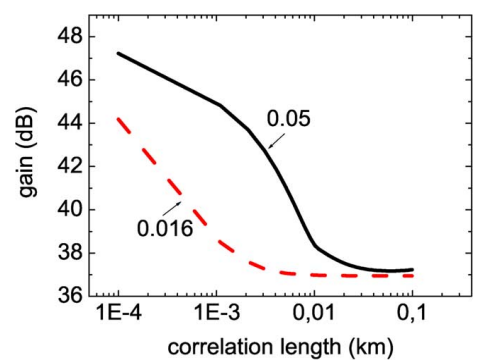

Fig. 5. Gain of a Raman polarizer as function of the correlation length. The SOP of the pump beam is $(1,0,0)$, the signal beam is scrambled. Values of the beat length $L_{B}$ are indicated on the plot in $\mathrm{km}$. Other parameters are the same as in Fig. 2, except that the input signal power is $1 \mu \mathrm{W}$.

Raman polarizers, the PMD coefficient is defined as in [13], $D_{p}=2 \sqrt{2} \pi\left(\sqrt{L_{c}}\right) /\left(L_{B}\right) \omega_{s}^{-1}$.

Finally, let us consider the gain characteristics of Raman polarizers. Owing to their strong polarization-dependent gain, the gain of a Raman polarizer is sizably larger than that of typical polarization insensitive Raman amplifiers (i.e., with high PMD values or that use polarization scrambled pump beams), see Fig. 5. This increase in gain demonstrates that Raman polarizers are simultaneously very efficient Raman amplifiers. This feature means that the amplification of signal beams with powers in the $\mathrm{mW}$ range will almost certainly lead to the depletion of the pump. This regime is left aside in this study, as our goal was the demonstration of the basic properties of Raman polarizers only. The regime with depleted pump is so rich and parameter-sensitive that it becomes rather difficult to draw general conclusions. However, if necessary our theory is capable of treating the depleted regime too.

\section{CONCLusion}

We have developed a general theory for describing the interaction of two beams in randomly birefringent fibers via the Kerr and Raman effects. This theory can be applied to both the co-propagation and counter-propagation configurations. Here we considered only the counter-propagating geometry, while details on the co-propagating regime can be found in [7]. A comparison between these two studies shows that the counter-propagating case is more demanding, in that it requires for instance PMD coefficients below $0.008 \mathrm{ps} / \sqrt{\mathrm{km}}$ for getting DOP slightly above 0.9 in a $2 \mathrm{~km}$ fiber. Whereas the co-propagating geometry can provide DOPs as high as 0.99 for a $D_{p}$ as large as 0.014 $\mathrm{ps} / \sqrt{\mathrm{km}}$ with only a $1.5 \mathrm{~km}$ long fiber. In both cases the pump power is $8 \mathrm{~W}$.

However, it is important to point out that the counter-propagating configuration has the advantage of clamping the signal SOP to the SOP of the pump source. In the co-propagating geometry the output signal SOP is also related to the pump SOP, but both depend on the stochastically changing birefringence of the fiber, thus complicating the control over the output signal SOP.

In addition to the theoretical model of the Raman amplification in randomly birefringent fibers, we presented the scheme for the quantification of the performances of Raman polarizers. We have identified three main characteristics of Raman polarizers: the DOP of the outcoming signal beam, its SOP defined in relation with the pump SOP, and the amplifier gain. The present 
study was limited to the undepleted regime only, although the theory is readily applicable to the depleted regime as well.

\section{ACKNOWLEDGMENT}

The authors would like to thank L. Palmieri and M. Santagiustina for valuable comments.

\section{REFERENCES}

[1] C. Headley and G. P. Agrawal, Raman Amplification. Amsterdam. New York: Elsevier, 2005.

[2] R. Hellwarth, J. Cherlow, and T.-T. Yang, "Origin and frequency dependence of nonlinear susceptibilities of glasses," Phys. Rev. B, vol. 11, pp. 964-967, 1974.

[3] R. H. Stolen, "Polarization effects in fiber Raman and Brillouin lasers," IEEE J. Quantum Electron., vol. QE-15, no. 10, pp. 1157-1160, Oct. 1979.

[4] D. J. Dougherty, F. X. Kartner, H. A. Haus, and E. P. Ippen, "Measurement of the Raman gain spectrum of optical fibers," Opt. Lett., vol. 20, no. 1 , pp. 31-33, 1995.

[5] M. Alfaid, D. van den Borne, T. Wuth, M. Kuschnerov, M. B. Astruc, P. Sillard, and H. de Waardt, "Long-haul optical transmission using 111-Gb/s polarization-multiplexed DQPSK modulation," presented at the 2010 Photonics Society Summer Topical Meeting Series, 2010.

[6] M. Martinelli, M. Cirigliano, M. Ferrario, L. Marazzi, and P. Martelli, "Evidence of Raman-induced polarization pulling," Opt. Exp., vol. 17, pp. 947-955, 2009.

[7] V. V. Kozlov, J. Nuño, J. D. Ania-Castañón, and S. Wabnitz, "Theory of fiber optic Raman polarizers," Opt. Lett. vol. 35, pp. 3970-3972, 2010 [Online]. Available: http://arxiv.org/abs/1009.1079

[8] L. Ursini, M. Santagiustina, and L. Palmieri, "Raman nonlinear polarization pulling under pump depletion regime in randomly birefringent fibers," IEEE Photon. Technol. Lett., to be published.

[9] Q. Lin and G. P. Agrawal, "Polarization mode dispersion induced fluctuations during Raman amplifications in optical fibers," Opt. Lett., vol. 27, no. 24, pp. 2194-2196, 2002.

[10] Q. Lin and G. P. Agrawal, "Vector theory of stimulated Raman scattering and its applications to fiber-based Raman amplifiers," J. Opt. Soc. Amer. B, vol. 20, no. 8, pp. 1616-1631, 2003.

[11] S. V. Sergeyev, S. Yu Popov, and A. T. Friberg, "Modeling polarization-dependent gain in fiber Raman amplifiers with randomly varying birefringence," Opt. Commun., vol. 262, p. 114, 2006.

[12] A. Galtarossa, L. Palmieri, M. Santagiustina, and L. Ursini, "Polarized backward Raman amplification in randomly birefringent fibers," J. Lightw. Technol., vol. 24, no. 11, pp. 4055-4063, Nov. 2006.

[13] P. K. A. Wai and C. R. Menyuk, "Polarization mode dispersion, decorrelation and diffusion in optical fibers with randomly varying birefringence," J. Lightw. Technol., vol. 14, no. 2, pp. 148-157, Feb. 1996.

[14] S. Pitois, G. Millot, and S. Wabnitz, "Nonlinear polarization dynamics of counterpropagating waves in an isotropic optical fiber: Theory and experiments," J. Opt. Soc. Amer. B., vol. 18, no. 4, pp. 432-443, 2001.

[15] S. Pitois, A. Picozzi, G. Millot, H. R. Jauslin, and M. Haelterman, "Polarization and modal attractors in conservative counterpropagating four-wave interaction," Europhys. Lett., vol. 70, no. 1, pp. 88-94, 2005.

[16] S. Pitois, J. Fatome, and G. Millot, "Polarization attraction using counterpropagating waves in optical fiber at telecommunication wavelengths," Opt. Exp., vol. 16, no. 9, pp. 6646-6651, 2008.

[17] E. Assémat, S. Lagrange, A. Picozzi, H. R. Jauslin, and D. Sugny, "Complete nonlinear polarization control in an optical fiber system," Opt. Lett., vol. 35, no. 12, pp. 2025-2027, 2010.

[18] J. Fatome, S. Pitois, P. Morin, and G. Millot, "Observation of light-bylight polarization control and stabilization in optical fibre for telecommunication applications," Opt. Exp., vol. 18, no. 15, pp. 15311-15317, 2010.

[19] V. V. Kozlov, J. Nuño, and S. Wabnitz, "Theory of lossless polarization attraction in telecommunication fibers," J. Op. Soc. Am. B, vol. 28, no. 1, pp. 100-108, 2011.

[20] J. Fatome, P. Morin, S. Pitois, and G. Millot, "Light-by-light polarization control of 10-Gbit/s RZ and NRZ telecommunication signals," $J$. Sel. Top. Quantum Electron., 2011, to be published.

[21] V. V. Kozlov and S. Wabnitz, "Theoretical study of polarization attraction in high-birefringence and spun fibers," Opt. Lett., vol. 35, no. 23, pp. 3949-3951, 2010.
[22] S. Pitois, A. Sauter, and G. Millot, "Simultaneous achievement of polarization attraction and Raman amplification in isotropic optical fibers," Opt. Lett., vol. 29, no. 6, pp. 599-601, 2004.

[23] C. R. Menyuk and B. S. Marks, "Interaction of polarization mode dispersion and nonlinearity in optical fiber transmission systems," $J$. Lightw. Technol., vol. 24, no. 7, pp. 2806-2826, Jul. 2006.

Victor V. Kozlov was born in Leningrad, USSR (now St. Petersburg, Russia), in 1968. He received the M.Sc. degree in physics and the Ph.D. degree in laser physics in 1992 and 1995, respectively, from the St. Petersburg University, St. Petersburg, Russia. In 2009 he received the degree of Doctor of Sciences from the Supreme Attestation Committee in Moscow.

In 1996, he joined the Institute of Physics (now Department of Physics) of the St. Petersburg University as a Research Scientist, and was promoted to Senior Research Scientist in 1998, and where he has become an Associate Professor in 2010. Meanwhile, he developed his scientific and teaching skills during long periods of stay with the Physics Department of Texas A\&M University, College Station; Rochester Theory Center of the University of Rochester, Rochester, NY; the Max Planck Institut fur Quantenoptik, Garching, Germany; and Abteiling fuer Quantenphysik, Universitaet Ulm, Germany. His main research interests are in ultrafast quantum optics, noise in fiber-optical (classical as well as quantum) communications, mode-locked lasers, and polarization effects in nonlinear fiber optics.

Javier Nuño was born in Aranda de Duero, Spain in 1983. He received the telecommunications degree from Universidad de Valladolid, Spain, in 2007 and the Master degree in laser technology from Universidad Politcnica de Madrid, Spain, in 2009. During 2007, he had a long stay in the Photonics Research Group at Aston University. Since 2009, he has been working towards the Ph.D. degree at Instituto de Óptica, CSIC, Madrid, Spain.

His current research interests include the exploitation of nonlinear effects in optical systems.

Juan Diego Ania-Castañón was born in Oviedo, Spain, in 1973. He received the M.Sc. degree in physics from Universidad Complutense de 1 Madrid, Spain, and the Ph.D. degree in theoretical physics from Universidad de Oviedo and Instituto de Estructura de la Materia, CSIC, Madrid, Spain.

In 2001 he joined the Photonics Research Group at Aston University, Birmingham, U.K., first as a Contract Research Fellow and then as an EPSRC Advanced Research Fellow from 2004. In 2007 he took an appointment as Tenured Scientist at Instituto de Optica, CSIC, Madrid, Spain. His main research interests include the study of nonlinear dynamics in optical systems and the exploitation of nonlinear effects in optical fiber.

Stefan Wabnitz received the Laurea degree in electronics engineering from the University of Rome "La Sapienza" in 1982, the M.S. degree in electrical engineering from the California Institute of Technology, in 1983, and the Ph.D. degree in applied electromagnetism from the Italian Ministry of Education in 1988.

He has been a Researcher of the Optical Communications Department of the Ugo Bordoni Foundation between 1985 and 1996, where he has contributed the theory of nonlinear wave propagation in optical fibers and waveguides, with particular interest in wave instabilities and soliton phenomena. In 1996 he became a full Professor in Physics at the University of Burgundy, Dijon, France. Between 1999 and 2003 he has been on leave from the University in order to carry out research and development in the telecommunication industry. He spent first two years working on optical soliton effects for submarine system applications at the Alcatel Research and Innovation Labs in Marcoussis, France, and two years as Manager of the Advanced Technology Group of Xtera Communications in Texas. Since September 2007 he has been a full Professor the Department of Electronics for Automation of the University of Brescia, Italy. His current research activities involve nonlinear propagation effects in high-bit-rate optical communication systems and in all-optical information processing devices. $\mathrm{He}$ is the author and co-author of over 400 refereed papers and conference presentations.

Dr. Wabnitz is the Deputy Editor of Elseviers Optical Fiber Technology, and is a member of the Optical Society of America and of the IEEE-LEOS. In October 2008 he was nominated Fellow of the Optical Society of America. 